\title{
Diferenças sociodemográficas, clínicas e de tratamento entre idosos com doenças cardiovasculares do SUS e de convênios/particulares
}

\section{Socio-demographic, clinical and treatment differences among elderly with cardiovascular diseases of SUS and covenants/private \\ Diferencias sociodemográficas, clínicas y de tratamiento entre ancianos con enfermedades cardiovasculares del SUS y de convenios / particulares}

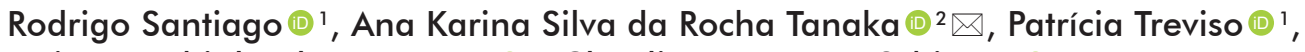
Daisy Zanchi de Abreu Botene ${ }^{1}$ ' , Claudine Lamanna Schirmer $\mathbb{1 0}^{3}$

' Centro Universitário Metodista IPA. Porto Alegre, RS, Brasil.

2 Universidade Federal do Rio Grande do Sul (UFRGS). Porto Alegre, RS, Brasil.

${ }^{3}$ Pontifícia Universidade Católica do Rio Grande do Sul (PUCRS). Porto Alegre, RS, Brasil.

\section{ARTICLE INFO}

\section{Article history}

Received: $31 / 08 / 2018$

Accepted: 28/03/2019

Published: 29/08/2019

\section{$\triangle$ Correspondent Author}

Ana Karina Silva da Rocha Tanaka

Escola de Enfermagem - UFRGS

Rua São Manoel 963, Santana

90620-001, Porto Alegre, RS, Brasil

ana.tanaka@ufrgs.br

\section{(C) 2019 All rights reserved}

\section{Editors}

Alfredo Cataldo Neto

Newton Luiz Terra

Assistant Editors

Paula Engroff

Vanessa Sgnaolin

\begin{abstract}
RESUMO
OBJETIVO: Avaliar as características sociodemográficas, clínicas e a prevalência de medicamentos utilizados por idosos com doenças cardiovasculares abordando a diferença entre usuários do sistema único de saúde (SUS) e de convênios/particulares durante a internação hospitalar.

MÉTODOS: Pesquisa transversal, retrospectiva, sendo conduzida em um hospital de grande porte de Porto Alegre, mediante revisão de prontuários dos pacientes internados nas unidades de internação coronariana no período compreendido entre janeiro e dezembro de 2015. A amostra foi constituída da avaliação de 152 prontuários, a coleta foi realizada através de um instrumento específico semiestruturado.

RESULTADOS: Total de 68 pacientes idosos pertenciam ao grupo de usuários do SUS. Os demais 84 prontuários eram usuários de diversos grupos de Convênios/Particulares. Em relação ao estilo de vida mostrou-se que o tabagismo foi representativo. Quanto aos medicamentos presentes no RENAME, o AAS $(94,1 \%$ vs $90,4 \%)$ e o Maleato de Enalapril $(73,5 \%$ VS $50,6 \%)$ foram, respectivamente, os mais citados pelos pacientes do SUS e convênios/particulares. Já o medicamento Atorvastatina Cálcica, fora do RENAME, foi prescrita em 73,8\% dos investigados do grupo SUS e 88,5\% de convênios/particulares.

CONCLUSÕES: Conclui-se que os pacientes do grupo convênios/particulares utilizam um número maior de medicamentos diferenciados que estão fora do relatório Nacional de Medicamentos Essenciais, do que os do grupo SUS. O grupo SUS se destaca por serem mais hipertensos e tabagistas e consequentemente realizam mais internações com patologias mais graves quando comparados ao outro grupo. Esses dados comparativos salientam a importância de ações que visem equilibrar as diferenças existentes entre as modalidades de planos de saúde atendidas no Brasil.
\end{abstract}

DESCRITORES: Doenças cardiovasculares; Utilização de medicamentos; Idosos. 


\section{ABSTRACT}

OBJECTIVE: To evaluate the sociodemographic, clinical characteristics and prevalence of medications used by elderly patients with cardiovascular diseases, addressing the difference between users of the single health care system (SUS) and private/covenant during hospitalization.

Methods: A retrospective cross-sectional study was carried out in a large hospital in Porto Alegre, by reviewing medical records of patients hospitalized at the coronary care units in the period from January to December 2015 . The sample consisted of the evaluation of 152 medical records, the collection was performed through a specific semi-structured instrument.

RESULTS: A total of 68 elderly patients belonged to the SUS user group. The other 84 medical records were users of several Covenants / Individuals groups. Regarding lifestyle, it was shown that smoking was representative. The presence of a significant association Regarding the clinical profile, all patients in both groups were hospitalized in coronary care units.

CONCLUSIONS: It is concluded that the patients in the private / covenants group use a greater number of drugs outside the National Essential Medicines report than those in the SUS group. The SUS group stands out for being more hypertensive and smokers and as a result, they perform more hospitalizations with more severe pathologies when compared to the other group. These comparative data highlight the importance of actions that aim to balance the differences between the modalities of health plans served in Brazil.

KeYwORDS: Cardiovascular diseases; Drug utilization; Elderly.

\section{RESUMEN}

OBJETIVOS: Evaluar las características sociodemográficas, clínicas y la prevalencia de medicamentos utilizados por ancianos con enfermedades cardiovasculares abordando la diferencia entre usuarios del sistema único de salud (SUS) y de convenios / particulares durante la internación hospitalaria.

MÉTODOS: Investigación transversal, retrospectiva, siendo conducida en un hospital de gran porte de Porto Alegre, mediante revisión de prontuarios de los pacientes internados en las unidades de internación coronaria en el período comprendido entre enero y diciembre de 2015 . La muestra fue constituida de la evaluación de 152 prontuarios, la recolección fue realizada a través de un instrumento específico semi-estructurado.

RESULTADOS: Total de 68 pacientes ancianos pertenecían al grupo de usuarios del SUS. Los demás 84 prontuarios eran usuarios de diversos grupos de Convenios / Particulares. En relación al estilo de vida se mostró que el tabaquismo fue representativo. En cuanto a los medicamentos presentes en RENAME, el AAS $(94,1 \%$ vs 90,4\%) y el Maleato de Enalapril $(73,5 \%$ VS 50,6\%) fueron, respectivamente, los más citados por los pacientes del SUS y convenios / los individuos. El medicamento Atorvastatina Cálcica, fuera del RENAME, fue prescrita en el 73,8\% de los investigados del grupo SUS y el $88,5 \%$ de convenios / particulares.

CONCLUSIONES: Se concluye que los pacientes del grupo convenios / particulares utilizan un número mayor de medicamentos diferenciados que están fuera del informe Nacional de Medicamentos Esenciales, que los del grupo SUS. El grupo SUS se destaca por ser más hipertensos y tabaquistas y consecuentemente realizan más internaciones con patologías más graves cuando comparadas al otro grupo. Estos datos comparativos subrayan la importancia de acciones que busquen equilibrar las diferencias existentes entre las modalidades de planes de salud atendidas en Brasil.

PALABRAS CLAVE: Enfermedades Cardiovasculares; Utilización de medicamentos; Anciano.

\section{INTRODUÇÃO}

A taxa de longevidade das pessoas está em ascensão. Isso se deve, entre outras causas, ao avanço da tecnologia e a mudança de paradigma de tratamento curativo para uma visão mais preventiva. No entanto, as doenças cardiovasculares continuam sendo as maiores responsáveis pelos óbitos da população adulta. ${ }^{1}$

Entre os principais tratamentos para as doenças cardiovasculares está o medicamentoso. No entanto, ao levar em consideração que um mesmo indivíduo pode apresentar diversas complicações relacionadas a essa doença, ele se torna, por vezes, obrigado a ingerir um número significativo de medicamentos simultaneamente. ${ }^{2}$

Por ser uma doença onde os sintomas demoram a trazer consequências e limitações, seu tratamento acaba sendo, inicialmente, negligenciado pelo paciente. Essa dificuldade se agrava quando se trata de idosos, já que cerca de 20 a 50\% dos pacientes em geral não aderem ao tratamento. ${ }^{3}$
No Brasil e no mundo, o principal influenciador na escolha dos medicamentos mais modernos e a questão financeira, ou seja, o consumo está, em grande parte, ligado ao poder aquisitivo da população. Levando em consideração que aproximadamente 50\% dos pacientes não tem acesso à compra de medicamentos privados, apenas a população com maior renda utiliza medicamentos de última geração. ${ }^{4}$

A literatura científica descreve a doença cardiovascular como a primeira causa de óbitos em adultos, a dificuldade de idosos em aderir ao tratamento medicamentoso, o maior acesso aos fármacos pelos pacientes com maior renda e a maior porcentagem na taxa de mortalidade por doenças cardiovasculares entre pacientes de baixa classe social. ${ }^{5}$

No entanto, pesquisas relacionadas ao número de medicamentos utilizados por idosos em hospitais são escassas e, quando comparado seu uso no Sistema Único de Saúde (SUS) e na saúde suplementar, ainda obtemos muitas lacunas. ${ }^{5}$

O tratamento medicamentoso na recuperação de idosos acometidos por doenças cardiovasculares é de 
grande importância, desta forma a educação para o uso correto contribui para melhora dos usuários.

Esta pesquisa teve como objetivo avaliar as características sociodemográficas, clínicas e a prevalência de medicamentos utilizados por idosos com doenças cardiovasculares, abordando a diferença entre usuários do SUS e de convênios/particulares durante a internação hospitalar.

\section{MÉTODO}

Trata-se de uma pesquisa de levantamento de dados, de corte transversal, retrospectiva e com abordagem quantitativa. Essa pesquisa foi conduzida em um hospital de grande porte, com 230 leitos, especializada em cardiopatias cardiovasculares de Porto Alegre, mediante revisão de prontuários dos pacientes internados nas unidades de internação coronariana, tanto no grupo SUS quanto no grupo dos convênios/ particulares. A coleta de dados foi feita através da revisão de prontuários, para o qual se utilizou um roteiro elaborado, semi-estruturado pelos pesquisadores.

Como critérios de inclusão utilizou-se prontuários de pacientes com 60 anos ou mais, admitidos nas Unidades de Internação no período de 1\%/01/2015 e $31 / 12 / 2015$, portadores de doença isquêmica, angina instável ou submetidos a cirurgia de revascularização do miocárdio ou troca de gerador de marca-passo. Foram excluídos dessa pesquisa os prontuários cuja informação sobre os medicamentos utilizados não estava clara ou incompleta.

Foram também extraídos os nomes de todos os fármacos de administração oral utilizados para doença cardiovascular, descritos pelo RENAME.

A apresentação dos resultados ocorreu pela estatística descritiva - distribuição absoluta e relativa (n - \%), bem como, pelas medidas de tendência central (média e mediana) e de variabilidade (desvio padrão e amplitude), com estudo da distribuição de dados das variáveis contínuas pelo teste de Kolmogorov-Smirnov. Para a análise bivariada entre variáveis categóricas foi utilizado o teste Qui-quadrado de Pearson $\left(\chi^{2}\right)$ ou Exato de Fisher. E para as variáveis contínuas, na comparação entre dois grupos independentes foram aplicados os testes de t-Studentou de Mann Whitney U. Ainda em relação às variáveis contínuas, quando comparadas entre três ou mais grupos (patologias) foram empregados os testes de Análise de Variância (One Way) - Post Hoc Tukey ou o teste de KruskalWallys - Post Hoc - Dunn. Os dados foram analisados no programa Statistical Package for Social Sciences versão 20.0 (SPSS Inc., Chicago, IL, USA, 2008) para Windows, sendo que, para critérios de decisão estatística adotou-se o nível de significância de 5\%.
Esta pesquisa seguiu as normas da Resolução do Conselho Nacional de Saúde (CNS), de acordo com a resolução no 466/2012, visto que ocorreu preservação do anonimato dos participantes. Esta pesquisa foi aprovada via plataforma Brasil, comitê de ética da instituição que foi realizada a pesquisa e pelo comitê de ética da universidade. Número do CEP: 1.136.573 de CAEE:45006015.9.0000.5308.

\section{RESULTADOS}

Os resultados apresentados referem-se a uma amostra de 152 usuários avaliados com idades acima de 60 anos, analisados segundo o tipo de grupo: SUS, $44,7 \%(n=68)$ e convênio/particular 55,3\% $(n=84)$.

$\mathrm{Na}$ avaliação das características sociodemográficas quando comparadas ao convênio, o sexo apresentou uma distribuição semelhante, com predomínio do sexo masculino, tanto no SUS, 67,6\% ( $\mathrm{n}=46)$, quanto no grupo convênios/particulares, 64,3\% $(n=54)$. Nas informações antropométricas, não foram detectadas diferenças estatisticamente significativas no peso, bem como, na altura. Em relação ao estilo de vida o tabagismo foi representativo $(\mathrm{p}=0,006)$, onde os idosos atendidos pelo SUS representavam $22,1 \%$ dos casos $(n=15)$, ex-tabagista 38,2\% $(n=26)$; enquanto que, os investigados do grupo convênios/particulares a maioria $64,0 \%(n=54)$ nunca fumou. A HAS foi a condição clínica mais prevalente $(\mathrm{p}=0,033)$.

No que se refere às patologias foi detectada associação estatística significativa ( $\mathrm{p}<0,001)$, de forma que os investigados do SUS apresentavam patologias de Cardiopatia isquêmica com uso de Extracorpórea (Z95.1) em 50,0\% dos casos ( $\mathrm{n}=34)$, e Infarto Agudo do Miocárdio (I21) em 48,5\% (n=33). Em relação ao grupo convênios/particulares, a associação significativa ocorreu na Angina Instável (I20.0), 27,4\% ( $\mathrm{n}=23)$ (Tabela 1).

Em relação à evolução da internação, foi detectada associação estatisticamente significativa $(p=0,021)$, de forma que nos dois grupos a maioria dos idosos tiveram a evolução alta hospitalar sem o procedimento Cateterismo (Tabela 1).

Avaliando as características gerais da amostra, no que se refere a associação da patologia com o sexo, no grupo de pacientes convênios/particulares houve diferença significativa $(p=0,036)$ onde o sexo masculino apresentou uma maior frequência no grupo com angina, $78,3 \%(n=18)$, enquanto que, com o sexo feminino a associação ocorreu com as doenças Cardiopatia isquêmica, 44,0\% $(\mathrm{n}=11)$, e Infarto Agudo do Miocárdio, 37,9\% $(\mathrm{n}=11)$. A média de idade do grupo convênios/particulares $(p=0,024)$ foi 
significativa no grupo Troca de Gerador de Marcapasso $(75,5 \pm 7,4)$ quando comparada as médias das idades das outras doenças (Tabela 2).

No grupo SUS, o tempo de internação diferiu de forma significativa $(\mathrm{p}<0,001)$ entre as doenças Cardiopatia isquêmica $(19,7 \pm 10,9$; mediana: 18,5$)$ e Infarto Agudo do Miocárdio (6,5 44,3; mediana: $5,0)$. No grupo convênios/particulares, novamente o grupo com doença Cardiopatia isquêmica concentrou significativamente $(\mathrm{p}<0,001)$ o maior tempo de internação $(18,5 \pm 14,4$; mediana 15,0$)$ quando comparado aos demais grupos de doenças. Em relação a evolução da internação, no convênio SUS verificou-se que os pacientes da Cardiopatia isquêmica mostra- ram-se significativamente associados a alta hospitalar sem cateterismo, 97,1\% ( $\mathrm{n}=33)$, enquanto aqueles com Infarto do Miocárdio, à associação ocorreu com a alta com cateterismo, 75,8\% $(\mathrm{n}=25) \quad(\mathrm{p}<0,001)$ (Tabela 2).

Na comparação do peso entre as doenças, verificou-se que, no grupo SUS as médias não diferiram, no entanto no grupo convênios/particulares se configurou a diferença significativa $(\mathrm{p}=0,015)$ apontando que os pacientes com Angina Instável $(81,0 \pm 15,4)$ apresentavam média de peso significativamente mais elevada que aqueles com Infarto Agudo do Miocárdio $(72,0 \pm 11,6)$ e Troca de Gerador de Marcapasso $(68,0 \pm 10,1)$ (Tabela 3$)$.

Tabela 1. Características sociodemográficas, clínicas e de estilo de vida da amostra estudada quando comparados os grupos SUS e convênios/particular.*

\begin{tabular}{|c|c|c|c|c|c|}
\hline \multirow{2}{*}{ Variáveis } & \multicolumn{2}{|c|}{ SUS $(n=68)$} & \multicolumn{2}{|c|}{ Convênios/Particulares ( $n=84$ ) } & \multirow{2}{*}{$\mathbf{p}$} \\
\hline & $\mathbf{n}$ & $\%$ & $\mathbf{n}$ & $\%$ & \\
\hline \multicolumn{6}{|l|}{ Sexo } \\
\hline Masculino & 46 & 67,6 & 54 & 64,3 & \multirow{2}{*}{$0,664 \S$} \\
\hline Feminino & 22 & 32,4 & 30 & 35,7 & \\
\hline \multicolumn{6}{|l|}{ Faixa etária (anos) } \\
\hline 60 a 69 & 42 & 61,8 & 44 & 52,4 & \multirow{2}{*}{$0,246^{\S}$} \\
\hline 70 ou mais & 26 & 38,2 & 40 & 47,6 & \\
\hline \multicolumn{6}{|l|}{ Peso (kg) } \\
\hline Média $\pm D P(A m p l i t u d e)$ & \multicolumn{2}{|c|}{$77,3 \pm 13,2(50-108)$} & \multicolumn{2}{|c|}{$76,0 \pm 13,5(50,0-120,0)$} & $0,582^{¥}$ \\
\hline \multicolumn{6}{|l|}{ Altura (m) } \\
\hline Média ĐDP (Amplitude) & \multicolumn{2}{|c|}{$1,66 \pm 0,08(1,46-1,83)$} & \multicolumn{2}{|c|}{$1,67 \pm 0,09(1,45-1,89)$} & $0,653^{*}$ \\
\hline \multicolumn{6}{|l|}{ Tabagismo } \\
\hline Tabagista & 15 & 22,1 & 7 & 8,3 & \multirow{3}{*}{$0,006^{\S}$} \\
\hline Ex tabagista & 26 & 38,2 & 23 & 27,4 & \\
\hline Nunca fumou & 27 & 39,7 & 54 & 64,0 & \\
\hline \multicolumn{6}{|l|}{ HAS } \\
\hline Sim & 59 & 86,8 & 61 & 72,6 & \multirow[b]{2}{*}{$0,033^{\S}$} \\
\hline Não & 9 & 13,2 & 23 & 27,4 & \\
\hline \multicolumn{6}{|l|}{ Dislipidemia } \\
\hline $\operatorname{Sim}$ & 29 & 42,6 & 39 & 46,4 & \multirow{2}{*}{$0,743^{\S}$} \\
\hline Não & 39 & 57,4 & 45 & 53,6 & \\
\hline \multicolumn{6}{|l|}{ Diabetes Melitus } \\
\hline Sim & 31 & 45,6 & 30 & 35,7 & \multirow{2}{*}{$0,217^{\S}$} \\
\hline Não & 37 & 54,4 & 54 & 64,3 & \\
\hline \multicolumn{6}{|l|}{ Patologia principal agrupada } \\
\hline Cardiopatia Isquêmica (Z95.1) & 34 & 50,0 & 31 & 36,9 & \multirow{4}{*}{$<0,001$} \\
\hline Angina Instável (I20.0) & 1 & 1,5 & 23 & 27,4 & \\
\hline Infarto Agudo do Miocárdio (I21) & 33 & 48,5 & 29 & 34,5 & \\
\hline Insuficiência Mitral por IAM (I23.5) & - & & 1 & 1,2 & \\
\hline \multicolumn{6}{|l|}{ Internação (dias) a } \\
\hline Média \pm DP (Amplitude) & \multirow{2}{*}{\multicolumn{2}{|c|}{$\begin{array}{c}13,0 \pm 10,6(1-57) \\
9,0(5,0-18,7)\end{array}$}} & \multirow{2}{*}{\multicolumn{2}{|c|}{$\begin{array}{c}11,3 \pm 10,3(1-64) \\
8,0(6,0-14,7)\end{array}$}} & \\
\hline Mediana ( $1^{\circ}-3^{\circ}$ Quartil) & & & & & 0,377 \\
\hline \multicolumn{6}{|l|}{ Evolução da internação } \\
\hline Alta Hospitalar sem Cateterismo & 40 & 58,8 & 63 & 75,0 & \\
\hline Alta Hospitalar com Cateterismo & 27 & 39,7 & 17 & 20,2 & $0,021^{\text {z }}$ \\
\hline Óbito & 1 & 1,5 & 4 & 4,8 & \\
\hline
\end{tabular}

* Percentuais obtidos com base no total de convênio.

a Variável com distribuição assimétrica (Kolmogorov-Smirnov, p<0,05); ${ }^{\S}$ Teste Qui-quadrado de Pearson; ${ }^{\mathfrak{z}}$ Teste Exato de Fisher (Simulação de Monte Carlo); ${ }^{¥}$ Teste t-Student para grupos independentes assumindo homogeneidade de variâncias; ‘ Teste de Mann Whitney. 
Tabela 2. Distribuição absoluta e relativa para sexo, faixa etária, evolução da internação; e medidas tendência central e de variabilidade para idade e tempo de internação, segundo o grupo de doença nos pacientes atendidos pelo SUS e convênios/particulares.

\begin{tabular}{|c|c|c|c|c|c|c|c|c|c|c|}
\hline \multirow[b]{2}{*}{ Variáveis } & \multicolumn{5}{|c|}{ SUS $(n=68)$} & \multicolumn{5}{|c|}{ Convênios/particulares (n=84) } \\
\hline & $\begin{array}{l}\text { Cardiopatia } \\
\text { isquêmica } \\
\text { com uso de } \\
\text { Extracorpórea } \\
(\mathrm{Z95.1})(\mathrm{n}=34)\end{array}$ & $\begin{array}{c}\text { Angina } \\
\text { Instável } \\
(I 20.0)(n=1)\end{array}$ & $\begin{array}{c}\text { Infarto } \\
\text { Agudo do } \\
\text { Miocárdio } \\
\text { (I21) }(\mathrm{n}=33)\end{array}$ & $\begin{array}{c}\text { Troca de } \\
\text { Gerador de } \\
\text { Marcapasso } \\
(\mathbf{I 2 0 . 0 )}(\mathrm{n}=\mathbf{0})\end{array}$ & $\mathbf{p}$ & $\begin{array}{l}\text { Cardiopatia } \\
\text { isquêmica } \\
\text { com uso de } \\
\text { Extracorpórea } \\
(\mathrm{Z95.1})(\mathrm{n}=\mathbf{2 5})\end{array}$ & $\begin{array}{c}\text { Angina } \\
\text { Instável } \\
(I 20.0)(n=23)\end{array}$ & $\begin{array}{l}\text { Infarto Agudo } \\
\text { do Miocárdio } \\
\text { (I21) }(\mathrm{n}=29)\end{array}$ & $\begin{array}{c}\text { Troca de } \\
\text { Gerador de } \\
\text { Marcapasso } \\
(\text { I20.0) }(n=6)\end{array}$ & p \\
\hline \multicolumn{11}{|l|}{ Sexo * } \\
\hline Masculino & $21(61,8)$ & $1(100,0)$ & $24(72,7)$ & - & $0,126^{\S}$ & $14(56,0)$ & $18(78,3)$ & $18(62,1)$ & $3(50,0)$ & $0,036^{\S}$ \\
\hline Feminino & $13(38,2)$ & - & $9(27,3)$ & - & & $11(44,0)$ & $5(21,7)$ & $11(37,9)$ & $3(50,0)$ & \\
\hline \multicolumn{11}{|l|}{ Idade (anos) } \\
\hline Média $\pm D P$ (Amplitude) & $\begin{array}{c}66,8 \pm 5,5 \\
(60-83)\end{array}$ & 69,0 & $\begin{array}{c}70,4 \pm 8,3 \\
(60-85)\end{array}$ & - & $0,201^{\epsilon}$ & $\begin{array}{l}69,1 \pm 4,9 \\
(61-78) b\end{array}$ & $\begin{array}{c}71,7 \pm 9,1^{\text {a }} \\
(60-87)\end{array}$ & $\begin{array}{c}69.7 \pm 8,7^{\epsilon} \\
(60-93)\end{array}$ & $\begin{array}{c}75,5 \pm 7,4^{\mathrm{a}} \\
(64-83)\end{array}$ & $0,024 \mathrm{~T}$ \\
\hline \multicolumn{11}{|l|}{ Faixa etária (anos)* } \\
\hline 60 a 69 & $24(70,6)$ & $1(100,0)$ & $17(51,5)$ & - & $0,102^{\S}$ & $15(60,0)$ & $10(43,5)$ & $17(58,6)$ & $1(16,7)$ & $0,112^{\S}$ \\
\hline 70 ou mais & $10(29,4)$ & - & $16(48,5)$ & - & & $10(40,0)$ & $13(56,5)$ & $12(41,4)$ & $5(83,3)$ & \\
\hline \multicolumn{11}{|l|}{ Internação (dias) ${ }^{\mathrm{a}}$} \\
\hline Média \pm DP (Amplitude) & $\begin{array}{c}19,7 \pm 10,9 \\
(4-57)\end{array}$ & $1(100,0)$ & $\begin{array}{c}6,5 \pm 4,3 \\
(1-18)\end{array}$ & - & $<0,001$ & $\begin{array}{c}18,5 \pm 14,4 \\
(3-64)\end{array}$ & $\begin{array}{c}8,1 \pm 7,5 \\
(1-29)\end{array}$ & $\begin{array}{c}8,9 \pm 4,9 \\
(3-22)\end{array}$ & $\begin{array}{c}5,8 \pm 3,6 \\
(1-10)\end{array}$ & $<0,001^{\mathrm{E}}$ \\
\hline Mediana ( $1^{\circ}-3^{\circ}$ Quartil) & $18,5(11,7-23,0)$ & - & $5,0(3,0-8,5)$ & - & & $15,0(9,0-22,00$ & $6,0(3,0-12,0)$ & $7,0(6,0-11,5)$ & $5,0(3,2-10,0)$ & \\
\hline \multicolumn{11}{|l|}{ Evolução da internação* } \\
\hline Alta Hospitalar sem Cateterismo & $33(97,1)$ & - & $7(21,2)$ & - & & $22(88,0)$ & $15(65,2)$ & $20(69,0)$ & $6(100,0)$ & \\
\hline Alta Hospitalar com Cateterismo & $1(2,9)$ & $1(100,0)$ & $25(75,8)$ & - & $<0,001^{3}$ & $1(4,0)$ & $7(30,4)$ & $9(31,0)$ & - & $0,144^{3}$ \\
\hline Óbito & - & - & $1(3,0)$ & - & & $2(8,0)$ & $1(4,3)$ & - & - & \\
\hline
\end{tabular}

* Resultados apresentados da forma n(\%), com percentual obtido com base no total de cada patologia.

${ }^{a}$ Variável com distribuição assimétrica (Kolmogorov-Smirnov, $\left.\mathrm{p}<0,05\right) ;{ }^{\circledR}$ Teste t-Student para grupos independentes assumindo heterogeneidade de variâncias; ${ }^{\circledR}$ Teste Qui-quadrado de Pearson; ${ }^{\top}$ Teste de Análise de Variância - One Way - Post Hoc Tukey; ${ }^{\mathrm{E}}$ Teste de kruskalWallys - Post Hoc Dunn; ${ }^{z}$ Teste Exato de Fisher (Simulação de Monte Carlo).

Tabela 3. Distribuição absoluta e relativa para tabagismo e HAS; e medidas tendência central e de variabilidade para peso e altura e segundo o grupo de doença em cada tipo de convênio.*

\begin{tabular}{|c|c|c|c|c|c|c|c|c|c|c|}
\hline \multirow[b]{2}{*}{ Variáveis } & \multicolumn{5}{|c|}{ SUS $(n=68)$} & \multicolumn{5}{|c|}{ Convênios/particulares $(\mathrm{n}=84)$} \\
\hline & $\begin{array}{c}\text { Cardiopatia } \\
\text { isquêmica } \\
\text { com uso de } \\
\text { Extracorpórea } \\
(\mathrm{Z95.1})(\mathrm{n}=34)\end{array}$ & $\begin{array}{c}\text { Angina } \\
\text { Instável } \\
(\mathbf{I 2 0 . 0 )}(\mathrm{n}=1)\end{array}$ & $\begin{array}{c}\text { Infarto } \\
\text { Agudo do } \\
\text { Miocárdio } \\
\text { (I21) }(\mathrm{n}=33)\end{array}$ & $\begin{array}{c}\text { Troca de } \\
\text { Gerador de } \\
\text { Marcapasso } \\
(\text { I20.0) }(n=0)\end{array}$ & $\mathbf{p}$ & $\begin{array}{c}\text { Cardiopatia } \\
\text { isquêmica } \\
\text { com uso de } \\
\text { Extracorpórea } \\
(Z 95.1)(n=25)\end{array}$ & $\begin{array}{c}\text { Angina } \\
\text { Instável } \\
(I 20.0)(n=23)\end{array}$ & $\begin{array}{c}\text { Infarto Agudo } \\
\text { do Miocárdio } \\
\text { (I21) }(n=29)\end{array}$ & $\begin{array}{c}\text { Troca de } \\
\text { Gerador de } \\
\text { Marcapasso } \\
(\mathbf{I 2 0 . 0 )}(\mathrm{n}=6)\end{array}$ & $\mathbf{p}$ \\
\hline \multicolumn{11}{|l|}{ Peso (kg) } \\
\hline Média $\pm \mathrm{DP}$ (Amplitude) & $\begin{array}{c}77,7 \pm 15,1 \\
(53-108)\end{array}$ & 96,0 & $\begin{array}{c}76,1 \pm 10,5 \\
(50-100)\end{array}$ & - & $0,477^{\epsilon}$ & $\begin{array}{c}77,7 \pm 12,7^{\text {a }} \\
(58-105)\end{array}$ & $\begin{array}{c}81,0 \pm 15,4^{\mathrm{a}} \\
(50-120)\end{array}$ & $\begin{array}{l}72,0 \pm 11,6^{\text {ez }} \\
(50,0-96,0)\end{array}$ & $\begin{array}{l}68,0 \pm 10,1^{3} \\
(56-83)\end{array}$ & $0,015^{\top}$ \\
\hline \multicolumn{11}{|l|}{ Altura (m) } \\
\hline Média $\pm \mathrm{DP}$ (Amplitude) & $\begin{array}{c}1,66 \pm 0,09 \\
(1,46-1,83)\end{array}$ & 1,65 & $\begin{array}{c}1,66 \pm 0,06 \\
(1,50-1,76)\end{array}$ & - & $0,682^{\epsilon}$ & $\begin{array}{c}1,66 \pm 0,08 \\
(1,45-1,89)\end{array}$ & $\begin{array}{c}1,69 \pm 0,09 \\
(1,50-1,87)\end{array}$ & $\begin{array}{c}1,66 \pm 0,08 \\
(1,45-1,79)\end{array}$ & $\begin{array}{c}1,62 \pm 0,07 \\
(1,52-1,70)\end{array}$ & $0,284 \mathrm{~T}$ \\
\hline \multicolumn{11}{|l|}{ Tabagismo } \\
\hline Tabagista & $4(11,8)$ & - & $11(33,3)$ & - & & $5(20,0)$ & & $2(6,9)$ & - & \\
\hline Ex tabagista & $16(47,1)$ & $1(100,0)$ & $9(27,3)$ & - & $0,255^{3}$ & $5(20,0)$ & $8(34,8)$ & $10(34,5)$ & - & $0,303^{3}$ \\
\hline Nunca fumou & $14(41,2)$ & - & $13(39,4)$ & - & & $15(60,0)$ & $15(65,2)$ & $17(58,6)$ & $6(100,0)$ & \\
\hline \multicolumn{11}{|l|}{ HAS } \\
\hline Sim & $33(97,1)$ & $1(100,0)$ & $25(75,8)$ & - & $0,094^{3}$ & $20(80,0)$ & $17(73,9)$ & $20(69,0)$ & $3(50,0)$ & $0,106^{3}$ \\
\hline Não & $1(2,9)$ & - & $8(24,2)$ & - & & $5(20,0)$ & $6(26,1)$ & $9(31,0)$ & $3(50,0)$ & \\
\hline
\end{tabular}

* Percentuais obtidos com base no total de convênio.

a Variável com distribuição assimétrica (Kolmogorov-Smirnov; $p<0,05)$; ${ }^{€}$ Teste t-Student para grupos independentes assumindo heterogeneidade de variâncias; ${ }^{\mathfrak{z}}$ Teste Exato de Fisher (Simulação de Monte Carlo); T Teste de Análise de Variância - One Way - Post Hoc Tukey.

De acordo com os resultados da Tabela 4, verificou-se que os medicamentos de uso contínuo mais utilizados no SUS e no grupo convênios/particulares foram o Ácido Acetilsalicílico e o Maleato de Enalapril. Os medicamentos de uso "se necessário" mais utilizados foram Dinitrato de Isossorbida e Captopril.

No que se refere aos medicamentos fora da RENAME, os casos concentraram-se no medicamento Atorvastatina Cálcica. 
Tabela 4. Distribuição absoluta e relativa para os medicamentos de uso contínuo, medicamentos necessários e fora da RENAME segundo o tipo de convênio.*

\begin{tabular}{|c|c|c|c|c|}
\hline \multirow{2}{*}{ Variáveis } & \multicolumn{2}{|c|}{ SUS $(n=68)$} & \multicolumn{2}{|c|}{ Convênios/particulares $(n=84)$} \\
\hline & $\mathbf{n}$ & $\%$ & $\mathbf{n}$ & $\%$ \\
\hline \multicolumn{5}{|l|}{ Medicamentos utilizados fixos } \\
\hline Ácido Acetilsalicílico & 64 & 94,1 & 75 & 90,4 \\
\hline Atenolol & 14 & 20,6 & 12 & 14,5 \\
\hline Besilato de Anlopidino & 12 & 17,6 & 13 & 15,7 \\
\hline Captopril & 2 & 2,9 & 1 & 1,2 \\
\hline Carvedilol & 8 & 11,8 & 21 & 25,3 \\
\hline Cloridrato de Amiodarona & 4 & 5,9 & 13 & 15,7 \\
\hline Cloridrato de Hidralazina & 4 & 5,9 & 4 & 4,8 \\
\hline Cloridrato de Verapamil & 1 & 1,5 & 2 & 2,4 \\
\hline Digoxina & 6 & 8,8 & 4 & 4,8 \\
\hline Dinitrato de Isossorbida & 0 & 0,0 & 1 & 1,2 \\
\hline Espironolactona & 7 & 10,3 & 7 & 8,4 \\
\hline Furosemida & 22 & 32,4 & 34 & 41,0 \\
\hline Hidroclorotiazida & 9 & 13,2 & 10 & 12,0 \\
\hline Losartana Potássica & 11 & 16,2 & 19 & 22,9 \\
\hline Maleato de Enalapril & 50 & 73,5 & 42 & 50,6 \\
\hline Mononitrato de Isossorbida & 41 & 60,3 & 35 & 42,2 \\
\hline Sinvastatina & 40 & 58,8 & 25 & 30,1 \\
\hline Succinato de Metoprolol & 27 & 39,7 & 37 & 44,6 \\
\hline Tartarato de Metoprolol & 23 & 33,8 & 20 & 24,1 \\
\hline Varfarina Sódica & 7 & 10,3 & 3 & 3,6 \\
\hline \multicolumn{5}{|c|}{ Medicamentos utilizados se necessário } \\
\hline Besilato de Anlopidino & 1 & 5,9 & 0 & 0,0 \\
\hline Captopril & 2 & 11,8 & 4 & 17,4 \\
\hline Dinitrato de Isossorbida & 16 & 94,1 & 19 & 82,6 \\
\hline Maleato de Enalapril & 0 & 0,0 & 1 & 4,3 \\
\hline \multicolumn{5}{|l|}{ Fora da RENAME } \\
\hline Atorvastatina Cálcica & 31 & 73,8 & 46 & 88,5 \\
\hline Ciprofibrato & 1 & 2,4 & 0 & 0,0 \\
\hline Clonidina & 8 & 19,0 & 7 & 13,5 \\
\hline Clortalidona & 1 & 2,4 & 1 & 1,9 \\
\hline Concor & 2 & 4,8 & 1 & 1,9 \\
\hline Diltiazen & 1 & 2,4 & 0 & 0,0 \\
\hline Duomo & 1 & 2,4 & 0 & 0,0 \\
\hline Felodipino & 0 & 0,0 & 1 & 1,9 \\
\hline Indapamida & 1 & 2,4 & 1 & 1,9 \\
\hline Moduretic & 0 & 0,0 & 1 & 1,9 \\
\hline Propatilnitrato & 2 & 4,8 & 3 & 5,8 \\
\hline Rosuvastatina & 3 & 7,1 & 0 & 0,0 \\
\hline Sotalol & 0 & 0,0 & 1 & 1,9 \\
\hline Ticagrelor & 2 & 4,8 & 3 & 5,8 \\
\hline Xarelto & 0 & 0,0 & 3 & 5,8 \\
\hline
\end{tabular}

* Percentuais obtidos com base no total de convênio; Teste Exato de Fisher (Simulação de Monte Carlo).

\section{DISCUSSÃO}

Em relação à prevalência de homens com doenças cardiovasculares, o resultado encontrado neste estudo está na média de outros já realizados, onde as médias ficaram entre $59,5 \%$ e $72 \%$ - dos indivíduos avaliados. Desta forma se observou a importância de estar educando a população em relação aos fatores modificáveis em relação ao risco para doenças cardiovasculares, entre eles excesso de peso e obesidade, ingestão de sal, ingestão de álcool, sedentarismo, fatores ambientais e fatores socioeconômicos. ${ }^{6-7}$
Alguns fatores se destacam e ajudam a mostrar o porquê dessa idade maior entre o grupo convênios/ particulares. $O$ pouco poder aquisitivo para compra de alimentos mais saudáveis entre usuários de baixa renda comprometeria até $145 \%$ da renda familiar se fosse usado para esse fim. ${ }^{8}$ Outro agravante é a vontade de se cuidar. Estudos concluem que desde adolescentes, os indivíduos de baixa renda não são tão preocupados com o corpo ideal, podendo ser este um agravante para a presença de doenças cardiovasculares futuras. ${ }^{9}$ Deve-se destacar também problemas no acesso aos leitos, onde a oferta é menor para pacientes do SUS 
quando comparados aos demais planos de saúde, comprometendo a equidade e, por consequência, sendo um fator preponderante na longevidade do grupo convênios/particulares. ${ }^{10}$

Esse número significativo de cateterismos no SUS em relação ao grupo convênios/particulares é diretamente proporcional ao número maior de cirurgias de revascularização do miocárdio realizadas por pacientes do Sistema Único de Saúde. Um dos fatores para essa cirurgia é a insuficiência coronariana, onde o cateterismo é uma das últimas alternativas a necessidade de revascularização. Segundo estudos, $51 \%$ das indicações de cateterismo cardíaco, dependentemente de plano de saúde, foram por insuficiência coronariana. ${ }^{11}$ Porém se estima que o SUS seja responsável por $80 \%$ dos cateterismos realizados no Brasil. ${ }^{12}$

Outros estudos mostram essa associação entre procedimentos mais complexos como motivos de internação no SUS quando comparado a outras modalidades de planos de saúde. A cardiopatia isquêmica é um procedimento realizado por pacientes cujas características sociodemográficas incluem a baixa escolaridade e a renda familiar de até um salário mínimo ${ }^{13}$, assim como o infarto agudo do miocárdio, onde estudos no atendimento pré-hospitalar mostram pacientes de baixa renda e escolaridade, que tiveram em sua maioria hospitais do SUS como destino. ${ }^{14}$

O valor elevado de medicamentos como Bupropiona e adesivo de nicotina são fatores que dificultam a cessação do tabagismo por parte de usuários de baixa renda. Talvez esse seja um motivo que explique a discrepância tão grande entre o número de indivíduos tabagistas no grupo SUS e no grupo convênios/ particulares. Estudos com diferentes populações, como pacientes cirúrgicos hospitalizados, ${ }^{15}$ pacientes psiquiátricos ${ }^{16}$ e até mesmo com uma população de moradores de residências escolhidas aleatoriamente ${ }^{17}$ apresentaram o mesmo resultado: associação do tabagismo com a baixa renda.

Resultado semelhante foi encontrado em outro estudo $^{18}$ onde $82,8 \%$ dos pacientes internados pelo SUS são hipertensos. A condição socioeconômica do indivíduo influencia a hipertensão até mesmo entre mais jovens e não miseráveis, como mostrou um estudo feito em Adventistas, onde indivíduos com renda familiar de 4 salários mínimos tinham índices de HAS melhores que os estudos gerais com pessoas de baixa renda, mas piores quando comparados a outro grupo de pessoas com renda familiar de 8 salários. ${ }^{19}$

A dislipidemia independe da classe econômica, ${ }^{20}$ estudos semelhantes mostram que tanto no grupo com melhores condições financeiras quanto naquele com as piores condições, o número de portadores de dislipidemia é quase idêntico. ${ }^{21}$ Desta forma independente de classe social a dislipidemia mostrase altamente prevalente e aumentada. A população portadora de altos níveis de dislipidemia, infelizmente não toma medidas de prevenção secundaria para o seu controle mesmo sabendo dos riscos. Sendo assim campanhas educativas e atividades de prevenção as dislipidemias são de extrema importância no RS.

Apesar de não haver grande diferença entre a associação do Diabetes Melitus entre os grupos estudados, o estudo mostra uma porcentagem considerável dessa comorbidade com a doença cardiovascular. Outras pesquisas mostram essa relação, tanto numa população de pacientes hipertensos, ${ }^{22}$ quanto naqueles que já tiveram um infarto o Diabetes é presente como uma das principais doenças associadas. ${ }^{23}$ A Diabetes Melitus a hipertensão arterial, o sedentarismo e a obesidade são um dos principais fatores de risco associado às doenças arteriais coronarianas, com altos índices de internações e custos elevados. Estes fatores podem ser controlados, com dietas, atividade física, mudanças comportamentais e com terapias farmacológicas.

O número superior de infarto ou cardiopatia isquêmica em pacientes do sexo feminino e do grupo convênios/particulares pode estar associado a uma maior prevalência de aterosclerose quando comparadas com o sexo masculino, sendo essa diferença mais significativa em países desenvolvidos. ${ }^{24}$ Salienta-se que mulheres têm hábitos sedentários mais rotineiros que os homens, como passar mais tempo sentadas e realizar menos atividade física. ${ }^{25}$

Estudo mostra valores semelhantes a média de idade dos pacientes submetidos a troca de gerador de marcapasso resultante desta pesquisa. ${ }^{26} \mathrm{No}$ entanto essa média tem grandes chances de aumentar gradativamente se levarmos em consideração que uma quantidade considerável de pacientes jovens a realizar o implante de marcapasso foi porque tiveram Doença de Chagas, tipo de patologia onde o número de casos novos era grande no passado e rara atualmente.

Apesar de o tempo médio de internação ser semelhante nos grupos SUS e convênios/particulares, essa permanência desde a admissão até a alta do paciente tem aumentado. Em pesquisa realizada no mesmo hospital, mas há 10 anos atrás, concluiu-se que o tempo de permanência de pacientes submetidos a revascularização do miocárdio era de 3 dias na Unidade pós-operatória e 13,49 dias na unidade de internação, totalizando um período médio de 2 a 3 dias inferior que o encontrado neste estudo. ${ }^{27}$

Percebe-se uma escolha pela cardiopatia isquêmica entre os pacientes do grupo convênios/particulares, 
onde apenas $31 \%$ dos diagnosticados com Infarto agudo do miocárdio foram submetidos a cateterismo, contra $75,8 \%$ dos do grupo SUS que tiveram diagnosticado a mesma patologia. Um estudo mostrou que 27\% dos pacientes submetidos a cateterismo necessitaram posteriormente passar pela cirurgia de revascularização do miocárdio. ${ }^{28}$

Sobre a estatística de maior peso em pacientes com angina instável, há grande relação com a idade menos avançada dos pacientes que apresentam essa patologia, que geralmente ocorre antes do infarto agudo do miocárdio e da troca de gerador de marcapasso. O idoso vai gradativamente reduzindo sua massa corporal magra, bem como a quantidade de água no organismo ${ }^{6}$. Como exemplo dessa perda de peso conforme a idade podemos citar um estudo onde dos idosos pesquisados, 36,7\% entre 60 e 69 anos eram obesos, contra 28,4\% dos de 70 a 79 anos e $14,6 \%$ daqueles a partir de 80 anos. $^{28}$

Dentre todas as causas de morte em idosos, as doenças cardiovasculares lideram. Neste estudo foi observada a prevalência de medicamentos utilizados por idosos com doenças cardiovasculares durante o período de internação hospitalar associado ao RENAME. Após definição do Ministério da Saúde, os medicamentos a serem contemplados pelo RENAME trouxeram inúmeras dificuldades para a manutenção do fornecimento de alguns medicamentos mais onerosos, desta forma muitos medicamentos utilizados para doenças cardiovasculares específicas, necessitam de justificativas médicas. Existem ainda algumas lacunas em relação ao fluxo de quantidade, duração do tratamento e padronização de medicamentos relacionados a doenças específicas, desta forma pode estar associado ao aumento dos dias de internação. ${ }^{29}$ Isso mostra a importância de se ter cada vez mais pesquisas relacionadas a esse tema na área da saúde, visando o gerenciamento do programa de medicamentos que vem sendo uma preocupação constante para os gestores do SUS e do setor privado. Foi possível observar diferenças, como o uso de medicamentos mais onerosos ou que estão fora da lista do RENAME com uma frequência maior nos pacientes do grupo convênios/particulares..$^{30}$ A literatura demonstra que a lista de medicamentos essenciais RENAME buscam a eficácia, qualidade e custo beneficio, porém muitos medicamentos para doença isquêmica do coração ainda permanecem em busca de evidências ou até mesmo semelhanças para o tratamento de doenças específicas, desta forma o mercado farmacêutico trás uma grande diversidade de produtos como padrão de competição focado em evidencias e novas tecnologias. ${ }^{31}$

Dentre as limitações do estudo foi evidenciada a falta de qualidade dos prontuários entre pacientes do SUS e Convênios/Particulares. Entretanto na análise específica de cada informação, os prontuários de pacientes de convênios/particulares tiveram maior qualidade em todas as variáveis. Um dos desafios do método de revisão de prontuários é a subestimação da especificação da qualidade dos dados registrados, observou-se que muitas vezes as informações importantes que foram realizadas muitas vezes deixaram lacunas em relação aos registros.

\section{CONSIDERAÇÕES FINAIS}

Podemos concluir que há uma diferença de perfil entre os pacientes do SUS e de convênios/particulares. O idoso com doença cardiovascular atendido no SUS em geral é uma pessoa mais velha, que interna por motivos mais graves e, por consequência, tem um tempo de internação maior. Isso se deve a um número maior de internações por patologias mais complexas como infarto agudo do miocárdio e cirurgia de revascularização do miocárdio. Os pacientes do grupo convênios/particulares por sua vez, acabam por ter um gasto maior, seja ele por internações mais longas, apesar da maioria ser por angina ou troca de gerador de marcapasso, ou por receber medicamentos mais caros.

Esse trabalho demostrou o quanto é importante o paciente idoso e seus familiares acompanharem todo o processo dos reais motivos que os levam a uma internação cirúrgica, bem como os se os tipos de tratamento medicamentoso são adequados perante suas patologias e comorbidades. A importância de realizar o processo preventivo em relação às doenças crônicas não transmissíveis pode estar auxiliando na prevenção de internações hospitalares.

Os medicamentos para doença cardiovascular são os mais consumidos pela população no mundo, e no que se refere a prevalência por faixa etária, os idosos lideram esse consumo. Entre os principais tratamentos para as doenças cardiovasculares está o medicamentoso. No entanto, ao levar em consideração que um mesmo indivíduo pode apresentar diversas complicações relacionadas a essa doença, ele se torna, por vezes, obrigado a ingerir um número significativo de medicações simultaneamente, desta forma a polimedicação ou polifarmácia é o maior risco de efeitos adversos e interações medicamentosas. Foi possível observar diferenças, como o uso de medicamentos mais onerosos ou que estão fora da lista do RENAME com uma frequência maior nos pacientes do grupo convênios/particulares.

As doenças cardiovasculares devem ser vistas com um olhar diferenciado pela equipe multidisciplinar, para verificar o melhor e mais adequado tratamento a ser 
realizado por ambos os grupos. Ao realizar um estudo comparativo entre dois grupos, a finalidade não foi a de definir qual o mais efetivo entre ambos, mas sim fazer com que aquele grupo que está com melhores índices sirva de exemplo para o outro, pois esse equilíbrio, quando alcançado, só tem a beneficiar aquele que é o principal foco dessa e de outras pesquisas na área da saúde: o nosso paciente.

\section{REFERÊNCIAS}

1. Magalhães APR, Paiva SC, Ferreira LOC, Aquino TA. A mortalidade de idosos no Recife: quando o morrer revela desigualdades. Epidemiol Serv Saúde. 2011;20(2):183-92. https://doi.org/10.5123/s1679-49742011000200007

2. Tavares NUL, Bertoldi AD, Thumé E, Facchini LA, França GVA, Mengue SS. Fatores associados à baixa adesão ao tratamento medicamentoso em idosos. Rev Saúde Pública. 2013;47(6):1092-101. https://doi. org/10.1590/s0034-89102013000901092

3. Silva RM, Caetano R. Gastos da Secretaria Municipal de Saúde do Rio de Janeiro, Brasil, com medicamentos: uma análise do período 2002-2011. Cad Saúde Pública. 2014;30(6):1207-18. https://doi.org/10.1590/0102$311 \times 00124612$

4. Sousa CV, Mesquita JMC, Lara JE. Análise da decisão de compra de medicamentos frente à existência de produtos substitutos: um estudo no município de Belo Horizonte, Brasil. Ciênc Saúde Coletiva. 2013;18(11):3311-20. https://doi.org/10.1590/s1413-81232013001100021

5. Mansur AP, Favarato D. Mortalidade por doenças cardiovasculares no Brasil e na região metropolitana de São Paulo: atualização 2011. Arq Bras Cardiol. 2012;99(2):755-61. https://doi.org/10.1590/s0066$782 \times 2012005000061$

6. Burmeister JE, Mosmann CB, Costa VB, Saraiva RT, Grandi RR, Bastos JP, Gonçalves LF, Rosito GA. Prevalence of cardiovascular risk factors in hemodialysis patients: The CORDIAL Study. Arq Bras Cardiol. 2014;102(5):473-80. https://doi.org/10.5935/abc.20140048

7. Faria TC, Giannini DT, Gasparini PVF, Rocha RM. Insuficiência cardíaca: relação entre parâmetros antropométricos, composição corporal e integridade celular. Int J Cardiovasc Sci. 2018;31(3):226-34.

8. Borges CA, Claro RM, Martins APB, Villar BS. Quanto custa para as famílias de baixa renda obterem uma dieta saudável no Brasil? Cad Saúde Pública. 2015;31(1): 137-48. https://doi.org/10.1590/0102-311x00005114

9. Tavares AF, Campana ANNB, Moraes MS. Apreciação corporal e modificação da aparência física em estudantes adolescentes de baixo poder aquisitivo. Psicol Esc Educ. 2012;16(2):275-82. https://doi.org/10.1590/s141385572012000200010

10. Machado JP, Martins M, Leite IC. O mix públicoprivado e os arranjos de financiamento hospitalar no Brasil. Saúde Debate. 2015;39(n. spe):39-50. https://doi. org/10.5935/0103-1104.2015s005245
11. Piegas LS, Feitosa G, Mattos LA, Nicolau JC, Rossi Neto JM, Timerman A, et al. IV Diretriz da Sociedade Brasileira de Cardiologia sobre tratamento do infarto agudo do miocárdio com supradesnível do segmento ST. Arq Bras Cardiol. 2009;93 (6 supl. 2):e179-e264. https://doi.org/10.1590/s0066-782x2001001400001

12. Piegas LS, Feitosa G, Mattos LA, Nicolau JC, Rossi Neto JM, Timerman A, et al. IV Diretriz da Sociedade Brasileira de Cardiologia sobre tratamento do Infarto agudo do miocárdio com supradesnível do segmento ST. Arq Bras Cardiol. 2009;93 (6 supl. 2):e179-e264. https://doi.org/10.1590/s0066-782x2001001400001

13. Lima TAS, Menezes TMO. Investigando a produção do conhecimento sobre a pessoa idosa longeva. Rev Bras Enferm. 2011;64(4):751-8. https://doi.org/10.1590/s003471672011000400019

14. Silva PRF, Cardoso CS, Ferreira HYS, Silva JC, Torres MTB, Silva RS, Chula ED. Avaliação do retardo préhospitalar no cuidado ao infarto agudo do miocárdio no Centro Oeste de Minas Gerais, Brasil. Rev Med Minas Gerais. 2015;25(3):353-62. https://doi.org/10.5935/22383182.20150070

15. Corrêa APA, Echer IC. Profile and motivation for smoking cessation in surgical inpatients. Rev Gaúcha Enferm. 2015;36(1):69-76. https://doi.org/10.1590/1983. 1447.2015 .01 .49133

16. Oliveira RM, Santos JLF, Furegato ARF. Perfil sociodemográfico de tabagistas internados em enfermaria psiquiátrica de hospital geral. Rev Bras Enferm. 2014; $67(3): 381-5$

17. Bortoluzzi MC, Kehrig RT, Loguercio AD, Traebertet JL. Prevalência e perfil dos usuários de tabaco de população adulta em cidade do Sul do Brasil (Joaçaba, SC). Ciênc Saúde Coletiva. 2011;16(3):1953-9. https://doi. org/10.1590/s1413-81232011000300029

18. Colósimo FC, Sousa AG, Silva GS, Piotto RF, Pierin AMG. Hipertensão arterial e fatores associados em pessoas submetidas à cirurgia de revascularização do miocárdio. Rev Esc Enferm USP. 2015;49(2):201-8. https://doi.org/10.1590/0080-6234199202600100017

19. Silva AL, Ribeiro AQ, Klein CH, Acurcio FA. Utilização de medicamentos por idosos brasileiros, de acordo com a faixa etária: um inquérito postal. Cad Saúde Pública. 2012;28(6):1033-45. https://doi.org/10.1590/s0102$311 \times 2012000600003$

20. Gus I, Ribeiro RA, Kato S, Bastos J, Medina C, Zazlavsky C, Portal VL, Timmers R, Markoski MM, Gottschall CAM. Variations in the prevalence of risk factors for coronary artery disease in Rio Grande do Sul-Brazil: a comparative analysis between 2002 and 2014. Arq Bras Cardiol. 2015;105(6):573-9. https://doi.org/10.5935/abc.20150127

21. Costa JSD, Reis MC, Silveira Filho CV, Linhares RS, Piccinini F, Fantinel E. Prevalência de internação hospitalar e fatores associados em Pelotas, RS. Rev Saúde Pública. 2010;44(5):923-33. https://doi.org/10.1590/s0034. 89102010005000026

22. Mamaniyujra CM, Avelar MCQ. Perfil epidemiológico de pacientes internados com infarto agudo do miocárdio: 
estudo retrospectivo. Rev Soc Cardiol Estado de São Paulo. 2012;22 (4 supl A):7-11 https://doi.org/10.29381/0103. $8559 / 2019290197-9$

23. Rodrigues ESR, Rezende AAB, Moreira RF, Souza JC, Pereira RO. Perfil dos fatores de risco cardiovascular em pacientes hipertensos. Rev Cereus. 2015;7(2):88-104. https://doi. org/10.18605/2175-7275/cereus.v7n2p88-104

24. Stefanini E, Brollo L. Seguimento ambulatorial do coronariopata crônico. Rev Soc Cardiol Estado de São Paulo. 2014;24(1):33-41.

25. Moraes SA, Checchio MV, Freitas ICM. Dislipidemia e fatores associados em adultos residentes em Ribeirão Preto, SP: resultados do Projeto EPIDCV. Arq Bras Endocrinol Metab. 2013;57(9):691-701. https://doi. org/10.1590/s0004-27302013000900004

26. Barros RT, Carvalho SMR, Silva MAM, Borges JBC. Avaliação de aspectos da qualidade de vida em pacientes pós-implante de marca-passo cardíaco. Rev Bras Cir Cardiovasc. 2014;29(1):37-44. https://doi. org/10.11606/d.22.2018.tde-05102018-181326

27. Morsch KT, Leguisamo CP, Camargo MD, Coronel CC, Mattos W, Ortiz LDN, Lima GG. Perfil ventilatório dos pacientes submetidos a cirurgia de revascularização do miocárdio. Rev Bras Cir Cardiovasc. 2009;24(2):180-7. https://doi.org/10.1590/s0102-76382009000200014

28. Sá MPBO, Lima LP, Rueda FG, Escobar RR, Cavalcanti PEF, Thé ECS, Escobar MAS, Lima RC. Estudo comparativo entre cirurgia de cardiopatia isquêmica com e sem circulação extracorpórea em mulheres. Rev Bras Cir Cardiovasc. 2010;25(2):238-44. https://doi.org/10.1590/ s0102-76382010000200018

29. Brasil. Conselho Nacional de Secretários de Saúde. Para entender a gestão do programa de medicamentos de dispensação em caráter excepcional. Brasília: CONASS; 2004. https://doi.org/10.21115/jbes.v8.n1.p39-46

30. Marsola APZC, Viana MAS, Nogueira MS, Hayashida M, Souza L, Cesarino EJ. Análise dos custos de medicamentos no tratamento das dislipidemias em Ribeirão Preto, São Paulo, Brasil. Rev Bras Cardiol. 2012;25(4):313-21.

31. Figueiredo TA, Schramm JMA, Pepe VLE. Seleção de medicamentos essenciais e a carga de doença no Brasil. Cad Saúde Pública. 2014;30(11):2344-56. https://doi. org $/ 10.1590 / 0102-311 \times 00165113$

\section{AUTHORS:}

RODRIGO SANTIAGO

Enfermeiro. Graduação em Centro Universitário Metodista IPA.

E-mail: digo.santiagodasilva@hotmail.com

Orcid: https://orcid.org/0000-0002-2198-2176

ANA KARINA SILVA DA ROCHA TANAKA

Enfermeira. Graduada pela Universidade do Vale do Rio dos Sinos - Unisinos. Mestrado em Gerontologia Biomédica na PUCRS. Dr를 em Gerontologia Biomédica na PUCRS. Prof ${ }^{a}$ Dr ${ }^{a}$ Escola de Enfermagem - na Universidade Federal do Rio Grande do Sul (UFRGS). Editora Associada da Revista Gaúcha de Enfermagem.

E-mail: ana.tanaka@ufrgs.br

Orcid: https://orcid.org/0000-0003-2488-3656
PATRÍCIA TREVISO

Enfermeira. Enfermeira. Graduada em Enfermagem pela Universidade Federal do Rio Grande do Sul - UFRGS, Mestrado e Doutorado em Ciências da Saúde pela Pontifícia Universidade Federal do Rio Grande do Sul - PUCRS. Coordenadora de Graduação do Centro Universitário Metodista IPA e Docente do curso de graduação em Enfermagem IPA.

E-mail: ptreviso15@gmail.com

Orcid: https://orcid.org/0000-0002-5015-6797

DAISY ZANCHI DE ABREU BOTENE

Enfermeira. Graduada em Enfermagem pela Universidade Federal do Rio Grande do Sul, Mestrado em Enfermagem - UFRGS, Doutorado em Enfermagem - UFRGS. Docente e coordenadora do Curso de Graduação em Enfermagem do Centro Universitário Metodista IPA (IPA). Membro do Comitê de Ética em Pesquisa e membro da Câmara de Pesquisa do Centro Universitário Metodista IPA (IPA).

E-mail: daisybotene@yahoo.com.br

Orcid: https://orcid.org/0000-0003-3702-1860

CLAUDINE LAMANNA SCHIRMER

Nutricionista graduada pela Pontifícia Universidade Católica do Rio Grande do Sul (PUCRS). Especialização em Saúde da Família e Comunidade pelo Programa de Residência Multiprofissional em Saúde (PREMUS) da PUCRS. Mestre e Doutoranda em Gerontologia Biomédica pelo Instituto de Geriatria e Gerontologia (IGG) da PUCRS.

E-mail: nutricionistaclaudine@gmail.com

Orcid: https://orcid.org/0000-0003-3803-740X 